\title{
RESUMEN
}

Las organizaciones están adoptando nuevos modelos de negocios enfocados a emprendimientos sustentables, es decir, más amigable con el planeta, el cual coacciona a una innovación en los nuevos ecosistemas emprendedores integrando una visión de una economía colaborativa y circular.

El propósito del presente estudio bibliométrico es examinar el estado de la cuestión del tema emprendimiento sustentable en los buscadores Scopus y Web of Science en los últimos cinco años.

Para ello, se utilizó una técnica bibliométrica con criterios de refinamientos y se obtuvo 1.168 documentos. Posteriormente, a través del software VOSviewer. se generaron mapas de densidad basados en la coocurrencia de los categorías.

En relación con los resultados obtenidos se considera que el emprendimiento sustentable es un tema joven aún, que posee un gran potencial de crecimiento y muchas empresas están integrando una planificación con orientación sustentable en sus procesos de producción y administrativos, permitiendo crear alianzas estrategias colaborativas con las cuales generan trabajos en conjunto para disminuir el desgaste de los recursos teniendo un enfoque triple en los aspectos económicos, sociales y ecológicos.

Palabras claves: emprendimiento sustentable, orientación, organizaciones.

\section{EMPRENDIMIENTO SUSTENTABLE COMO MODELO DE NEGOCIOS PARA LAS ORGANIZACIONES.}

Mtro. Jesús Humberto Lara Félix $/$ Dr. Juan Mejía Trejo ${ }_{2}$

\author{
Fechas de recepción: 15 de Junio de 2021 \\ Fecha de aceptación: 14 de Diciembre de 2021
}

DOl: http://doi.org/10.22370/riace.2021.10.1.2863

1 Centro Universitario de Ciencias Economicas-Administrativas, Universidad de Guadalajara, Zapopan, México, Imhumbertolara@gmail.com

2 Centro Universitario de Ciencias Economicas-Administrativas, Universidad de Guadalajara, Zapopan, México, juanmejiatrejo@hotmail.com 


\title{
USTAINABLE ENTREPRENEURSHIP AS A BUSINESS MODEL FOR ORGANIZATIONS.
}

\begin{abstract}
Organizations are adopting new business models focused on sustainable entrepreneurship, that is, more friendly to the planet, which coerces innovation in new entrepreneurial ecosystems by integrating a vision of a collaborative and circular economy.

The purpose of this bibliometric study is to examine the state of the question of sustainable entrepreneurship in the search engines Scopus and Web of Science over the past five years.

For this, a bibliometric technique was used with refinement criteria and 1,168 documents were obtained. Later, through the VOSViewer software, density maps were generated based on the co-occurrence of the categories.

In relation to the results obtained, sustainable entrepreneurship is considered to be still a young topic, with great growth potential, and many companies are integrating sustainably oriented planning into their production and administrative processes, allowing the creation of partnerships and collaborative strategies with which generate joint work to reduce the wear and tear of resources by taking a threefold focus on economic, social and ecological aspects.
\end{abstract}

Keywords: sustainable entrepreneurship, guidance, organizations. 


\section{INTRODUCCIÓN}

En función del comportamiento consumista que ha mostrado la humanidad en las últimas cinco décadas, la atención de la sociedad y en especial de la comunidad científica, enciende la percepción al observar un mal comportamiento y uso de los recursos (Hrivnák et al., 2021).

Las empresas en los ultimos años, han perseguido un solo objetivo, captar mayor participación en los mercados para incrementar sus ventas y de esta manera sobrevivir en el mundo empresarial (Belz y Binder, 2017). En consecuencia, el perseguir este objetivo y centrarse unicamente en satisfacer a los consumidores diseñando y creando productos y servicios más personalizados, provocó un incremento en las tasas de obsolescencia programada en ellos, esto dio como resultado un mayor consumo de recursos que son extraídos del planeta (Gast et al., 2017).

Al implementar los recursos que proporciona el planeta de forma poco sustentable ha hecho que emergan problemas tanto para las organizaciones como para la humanidad. La sobreexplotación de los recursos en combinación con la contaminación, ha causado el desgaste de los mantos acuiferos, bosques, selvas, flora/fauna y extinción de ecosistemas naturales que fueron usados de modo desmedido y poco sustentable (Guta et al., 2017). Asimismo, mientras no respecte e invada la humanidad los espacios naturales y las experimente nuevas fuentes de alimentación para satisfacer la necesidad básica que es la alimentación pudiera brotar nuevas enfermedades coaccionar grandes problemas mundiales (Al Omoush et al., 2017).

Al denotar que la demanda de recursos tanto renovables como no renovables sobrepasa el nivel de restauración, permite evidenciar que los recursos que proporciona la tierra son finitos y no infinitos. En otras palabras, de mantener el mismo ritmo que ha mostrado la humanidad, muchos problemas tendrán las generaciones futuras (Chu et al., 2021). Problemas como contaminación, pérdida de recursos y desigualdad de desarrollo económico, son algunos que deben detenerse. Por tal motivo, en 1972 en la confrerencia de las naciones unidas (ONU), la cual fue efectuada en Estocolmo, se expuso las limitantes físicas del planeta, que de continuar de la manera que ha venido viviendo la humanidad, únicamente sobreexplotando los recursos naturales y el crecimiento demográfico, emergerán diversas problemáticas (Joo Lee, 2019).

Al poner sobre la mesa la importancia del cuidado del medio ambiente la ONU comenzó un estudio que duró alrededor de tres años, los cuales fuerom comprendidos por foros, debates, discursos y audiencias públicas en todos los continentes relacionada con la falta de conciencia hacia los recursos del planeta. Al finalizar el período de estudio, la ONU publicó el documento "Nuestro futuro común", además, fue nombrado el informe de Brutland (Azam-Roomi et al., 2021). Dicho informe tiene como principal objetivo el desarrollo de la humanidad, pero 
de una manera equitativa e igualitaria con la sociedad, la economía y el ambiente, para cuidar la sobrevivencia de la misma y una de las conclusiones es que el desconocimiento del efecto que ocasiona el consumo de productos y servicios al término del periodo de uso, impacta en el planeta (Liu et al., 2021).

En México, fue hasta el 2007, en el sexenio del ex presidente Felipe Calderón Hinojosa que se integró mediante materias educativas relacionadas con la sustentabilidad, para formar mediante la educación mayor conciencia sobre los problemas sociales, económicas y ambientales (Ferreira Leite et al., 2021).

Este fenómeno, ha coaccionado nuevos enfoques y modelos de negocios, llevando a las organizaciones a una reestructuración nueva con criterios diferentes, teniendo en cuenta los aspectos sociales, economicos y ambientales. Asimismo, en la actualidad se ha presentado situaciones que han reforzado el origen de un emprendimiento sustentable por parte de las empresas nuevas y más longevas (Soo Sung y Park, 2018).

Aspectos como el cambio climático provocado por el hombre es un reto de vital importancia para la humanidad en el presente siglo XXI. Esto ha despertado en algunas empresas internacionales un nuevo modelo de negocios enfocado hacia una economía compartida (Slawinski et al., 2017). Una economía compartida es un modelo económico moderno que consiste en trabajar de manera colaborativa entre la persona que vende (entrega valor) y quien lo necesita (compra), es un trato directo en el cual la figura de los intermediarios desaparece, este modelo puede aplicarse mediante el trueque de productos, servicios equivalentes o transacciones comerciales en efectivo (Littlewood y Holt, 2018). Además, reconoce una estrategia de negocios compatible con el emprendimiento sustentable, de manera que la interacción comercial se da entre las personas que intercambian bienes equitativos para satisfacer las necesidades insatisfecha (Schulenkorf, 2017). El internet es un importante precursor de dicha economía, de esta manera, los consumidores tienen acceso a productos o servicios a un mejor precio.

Con el pasar de los años, el acto de emprender se ha convertido en una acción más compleja y poco redituable para muchas personas en el mundo. Ejecutar el emprendimiento representa enfrentar retos como escenarios complejos, dinámicos e incluso disruptivos. En el cual los riesgos son eminentes y desafiar la incertidumbre representa parte de la vida y formación que enfrentan los emprendedores (Carayannis et al., 2017).

Sin embargo, esta situación se comenzó a intensificar a partir del surgimiento de un mundo globalizado en el año 1980 y el énfasis competitivo ocasionó que el emprendimiento se centrara en la creación de vinculos, agrupaciones corporativas, redes colaborativas que estimularán el aprendizaje entre los elementos de los sistemas empresariales (Schot y Steinmueller, 2018).

El emprendimiento sustentable se debe centrar en la necesidad tanto de la sociedad como los que representan la acción ejercida hacia el planeta (Theodoraki et al., 
2018). A pesar de que el tema es muy jóven y poco explorado aún, esto representa una gran oportunidad para la humanidad, así como una manera de impactar lo menos posible a los ecosistemas, recursos, flora/fauna y especies animales (Skuratovych et al., 2021). El fundamento se centra en la teoría del comportamiento planificado, en el cual los individuos basan la conducta en actitudes, normas subjetivas y control conductual, que fungen como impulsores positivos/negativos y representativos para la intención de emprendimiento sustentable. Por tanto, une varios elementos; los cuales estan conformados por la empresa, el planeta y la sociedad en general (Hall et al., 2010).

Las empresas que ejercen la sustentabilidad ven en ella tres conjuntos prácticos a un nivel micro: crear entornos más propicios, alterar los estándares normativos y reformular el estigma de la sustentabilidad. Asimismo, los emprendedores deben tener mayor involucramiento para alcanzar credibilidad y posicionamiento en una economía compartida (DiVito y Bohnsack, 2017). La acción de los emprendedores ayudan a fundamentar y defender la sustentabilidad en las organizaciones, de esta manera, mejoran la transición en los ecosistemas empresariales (Urbaniec, 2018).

Los ecosistemas empresariales sustentables están más fuertemente vinculados con el aspecto social en comparación con los aspectos económico y ecologicos que son las dimensiones del desarrollo sustentable (Demirel et al., 2019). No obstante, el emprendimiento social ha detectado cuatro nuevas dimensiones claves la social, económica, de comportamiento y gobernanza. Además, se basan en la teoría de cambio la que está fuertemente enlazada con el emprendimiento sustentable y social (Stubbs, 2017).

La sustentabilidad, innovación y emprendimiento muestran una fuerte interconexión. Estos nuevos enfoques dislumbran un nuevo camino para avanzar en estudios sobre las micro y macro empresas emergentes sustentables, las cuales crearon un cambio sistemático en la gestión de las relaciones innovadoras (Horisch et al., 2017).

Parte fundamental para llevar a cabo una gestión ambiental y sea adaptada por las empresas, es responsabilidad de los tomadores de decisiones, los cuales deben tener un pensamiento abierto a nuevos procesos y nuevas maneras de hacer las cosas en las organizaciones, pues son los que tienen la última palabra para adaptar este nuevo modelo de negocios que posee un fuerte desempeño de la heterogeneidad de la empresa (Muralidharan y Pathak, 2018). El liderazgo sustentable da una gran diferencia que el convencional, tanto en las empresas emergentes como las ya establecidas como las que van a surgir (Vásquez-Bernal y Mosquera-Laverde, 2021).

Las actitudes que se deben considerar en el emprendimiento sustentable son el comportamiento y las motivaciones como impulsores en los clientes/consumidor final. Los emprendedores deben captar las oportunidades y necesidades de las dimensiones del desarrollo sustentable como el económico, social y ambiental, 
que se desprenden de los 17 objetivos del desarrollo sustentable establecidos por la ONU (Pietrzak et al., 2017). Estos adhieren un impacto positivo a largo plazo para el emprendimiento sustentable. Por otra parte, el emprendimiento tradicional que canaliza unicamente en la dimensión económica que a su vez se transfiere a la necesidad inmediata, ha desmotrado que este enfoque da como resultado un impacto negativo y poco rentable para muchas organizaciones (Ben Youssef et al., 2018)

La causa-efecto contemplada en un corto y largo plazo para que los emprendedores impulsen como una oportunidad a las dimensiones del desarrollo sustentable da para mucha investigación a largo plazo. En contraste, la oportunidad impulsada por la necesidad no tiene ningún efecto sobre las dimensiones que componen el desarrollo sustentable y no muestran mucha oportunidad de investigación a largo plazo (Luu, 2017).

En fechas recientes, ha surgido un interés por relacionar el emprendimiento con la sustentabilidad situación que ha ido adquiriendo mayor importancia mediante el conocimiento en el tema, y se ha creado la definición de emprendimiento sustentable de tal forma que ha brotado un umbral para el fomento de una economia social y ecológica asentada, que incentiva un crecimiento equitativo e inclusivo, esto ha llevado a las empresas a una reingenieria en la estructura organizacional (Muñoz y Cohen, 2018).

Asimismo, esto demanda un nuevo modelo de negocios para los emprendedores que deben renovarse de una manera disruptiva la eficacia de los recursos mendiante la eliminación de desperdicios y dejando el modelo lineal de hacer-usar-desechar atrás (Littlewood y Holt, 2018).

La aportación del emprendimiento sustentable es dar nuevas alternativas que ayuden a debilitar los problemas sociales y ambientales a través de negocios exitosos con fines de lucro (Schulenkorf, 2017) requiriendo la inversión de tiempo y esfuerzos para realizar nuevas innovaciones sustentables en las empresas y organizaciones, donde se procuren aplicar nuevos procesos que aminoricen el rápido desgaste de los recursos del planeta. Esta metodología debe seguirse tanto para las empresas ya establecidas con años participando en el mercado, como a las empresas que están por emerger (Yang et al, 2021).

El espíritu emprendedor convencional carece de una inclusión social y ecológica, ya que solo incluia cuestiones económicas que se pudiera considerar la más palpable al momento de meditar la acción de emprender. Esto muestra que el emprendimiento debe integrar una visión basada en recursos para dar mayor valor a la propuesta de valor que ofrece al mercado meta y que se traduzca en una ventaja competitiva para la empresa sustentable (Saebi et al., 2019).

Por tal motivo, el propósito del presente estudio bibliométrico es examinar el estado de la cuestión del tema emprendimiento sustentable en los buscadores Scopus y Web of Science en los últimos cinco años. 
La artículo está compuesto por metodología, resultados, discusión y conclusiones.

\section{METODOLOGÍA}

En palabras de Ayala-Picazo (2017) la técnica de bibliometría posee diversas bondades y beneficios para la ciencia, esta actividad permite recopilar información, aplicar un método de escrutinio y cuantificar diversos aspectos del fenomeno a estudiar, evidenciando los principales autores, documentos, formatos, idiomas y periodos de tiempo con mayor productividad.

Para concretar esta metodología, se indagó en las bases de datos Scopus y Web of Science, pues son consideradas como fuentes importantes y confiables para la comunidad cientifíca. Al ser motores de busqueda amplios contienen desde artículos, libros y memorias de congresos, por tal motivo, suministra la información necesaria para enriquecer el conocimiento a nivel mundial. Actualmente, son multidisciplinarias, en las que se puede encuentrar diversos campos de las ciencias tales como medicina, ciencias sociales, negocios y psicologia y humanidades. De igual manera, tienen la funcionalidad de extraer datos que son recolectados en formato de excel con extensión .CSV que también es compatible con el software VOSviewer que posee la aplicación de crear mapas correlacionables.

La búsqueda de información se llevó a cabo del 24 al 26 y del 30 al 31 de mayo del 2021, para extraer documentos finales el día 01 de Junio del mismo año. Se realiza en el idioma inglés y se afectuaron una serie de refinamientos que ayudaron a ordenar los documentos por palabras claves, área y tiempo en ambas bases de datos. El primer refinamiento fue la elección de las palabras clave que fueron "entrepreneurship" (emprendimiento) AND "sustainability" (sustentabilidad), se delimitó la coincidencia de estas palabras clave en el título, resumen y palabras clave de los documentos analizados. El resultado arrojó en Scopus 1.095 y Web Of Science 1.710 documentos. Posteriromente, se aplicó el criterio de refinamiento, por área temática; negocios, gestión, administración y contabilidad, dando como resultado en Scopus 1.095 y en Web Of Science 753. Por úlitmo, el tercer criterio de refinamiento, por línea de tiempo (2017-2021) con el que se obtuvo en Scopus 652 y Web Of Science 516 documentos.

Con estas cifras se elaboró el análisis del presente estudio bibliométrico, a pesar de que en su mayoria son artículos científicos, se contemplaron todo tipo de documentos que se encuentran en el tesauro de ambos buscadores, lo cual marca que los documentos citados en el presente estudio servirán para reforzar el conocimiento sobre el tema. Adicionlamente, se exportaron a un archivo excel con extensión .CSV para la organización y manipulación de los datos, así como la expresión de grafícas, mapas y tablas a través del software VOSviewer. 


\section{RESULTADOS}

Los resultados del estudio bibliomético en los buscadores Scopus y Web Of Science con los criterios de refinamiento, son los siguientes:

\section{Figura 1}

Publicación en Scopus y WoS

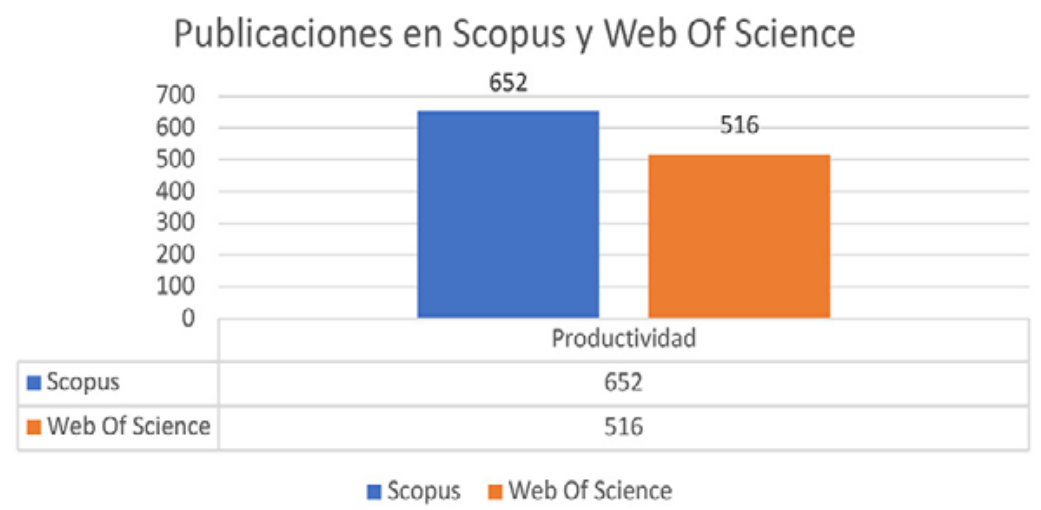

Fuente: Elaboración propia a partir de Scopus y WoS, 2021.

En la figura 1, se exponen los resultados finales de los criterios de refinamiento aplicados a los buscadores Scopus y Web Of Science, el buscador que más alberga documentos sobre el tema emprendimiento sustentable es Scopus con 652 documentos.

\section{Figura 2}

Publicación por año en Scopus y WoS

\section{Publicaciones del 2017-2021 en Scopus y WoS}

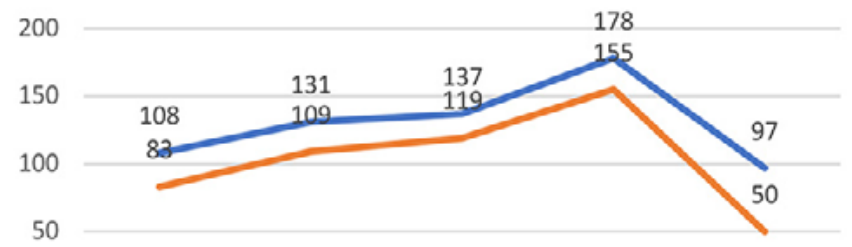

\begin{tabular}{|c|c|c|c|c|c|}
\hline \multicolumn{1}{c|}{0} & 2017 & 2018 & 2019 & 2020 & 2021 \\
\cline { 2 - 6 } & 108 & 131 & 137 & 178 & 97 \\
\hline Scopus & 83 & 109 & 119 & 155 & 50 \\
\hline
\end{tabular}

$\longrightarrow$ Scopus Web Of Science

Fuente: Elaboración propia a partir de Scopus y WoS, 2021. 
En la figura 2, se observa el comportamiento de publicación en el periodo 20172021. El año con mayor cantidad de publicaciones del tema emprendimiento sustentable fue el año 2020, con un total de 178 en Scopus y 155 en WoS.

\section{Tabla 1}

Publicaciones por países

\begin{tabular}{llcllc}
\hline $\begin{array}{l}\text { Scopus } \\
\text { N. }\end{array}$ & País & $\begin{array}{c}\text { Cantidad de } \\
\text { documentos }\end{array}$ & N. & País & $\begin{array}{c}\text { Cantidad de } \\
\text { Documentos }\end{array}$ \\
\hline 1 & Estados Unidos & 88 & 1 & Estados Unidos & 94 \\
2 & Reino Unido & 70 & 2 & Reino Unido & 65 \\
3 & Alemania & 68 & 3 & Alemania & 51 \\
4 & España & 39 & 4 & España & 38 \\
5 & Italia & 34 & 5 & Italia & 37 \\
6 & India & 32 & 6 & Canada & 31 \\
7 & Sur Africa & 31 & 7 & Francia & 28 \\
8 & Paises Bajos & 30 & 8 & India & 28 \\
9 & Francia & 23 & 9 & Australia & 24 \\
10 & Portugal & 23 & 10 & Paises Bajos & 23 \\
\hline
\end{tabular}

Fuente: Elaboración propia a partir de Scopus y WoS (2021).

En ambos buscadores, el país con mayor cantidad de publicaciones del tema emprendimiento sustentable es Estados Unidos con 88 en Scopus y 94 en WoS, según Tabla 1.

Parte importante para que un tema tenga mayor propagación en la comunidad cientifica es por el aporte de los investigadores que trabajan en tema en diferentes escenarios. A continuación, en la tabla 2, se presentan los principales autores que aparecen en ambos buscadores Scopus y WoS.

\section{Tabla 2}

Principales autores que escriben sobre Emprendimiento sustentable

\begin{tabular}{llcllc}
\hline $\begin{array}{l}\text { Scopus } \\
\text { N. }\end{array}$ & Autores & $\begin{array}{c}\text { Cantidad de } \\
\text { documentos }\end{array}$ & N. & Autores & $\begin{array}{c}\text { Cantidad de } \\
\text { Documentos }\end{array}$ \\
\hline 1 & Hörisch, J. & 7 & 1 & Kraus S. & 8 \\
2 & Fichter, K. & 6 & 2 & Schaltegger S. & 5 \\
3 & Dickel, P. & 5 & 3 & Halberstadt J. & 4 \\
4 & Jones, P. & 5 & 4 & Horisch J. & 4 \\
5 & Ratten, V. & 5 & 5 & Munoz P. & 4 \\
6 & Wagner, M. & 5 & 6 & Puumalainen K. & 4 \\
7 & Dressler, M. & 4 & 7 & Ratten V. & 4
\end{tabular}




\begin{tabular}{llllll}
8 & Moon, C. & 4 & 8 & Amankwah-amoah J. & 3 \\
9 & Muñoz, P. & 4 & 9 & Bals L. & 3 \\
10 & Passiante, G. & 4 & 10 & Cohen B. & 3 \\
\hline
\end{tabular}

Fuente: Elaboración propia a partir de Scopus y WoS, 2021.

El principal autor en el buscador Scopus es Hörisch, J. con 7 documentos publicados, seguido de Fichter, K. con 6 documentos y Dickel, P. con 5.

En el buscador WoS Kraus S. cuenta con 8 documentos publicados, seguido de Schaltegger S. con 5 y Halberstadt J. con 4 documentos.

Llama la atención que Horisch J. aparece en WoS con 4 documentos y en el buscador Scopus es el autor con mayor productividad con 7 .

En la universidades que más publican sobre el tema emprendimiento sustentable se encuentra Leuphana Universität Lüneburg en ambos buscadores (ver tabla 3).

\section{Tabla 3}

Principales Universidades que publican sobre Emprendimiento Sustentable

\begin{tabular}{|c|c|c|c|c|c|}
\hline \multicolumn{2}{|c|}{ Scopus } & \multicolumn{3}{|c|}{ Web Of Science } & \multirow[b]{2}{*}{$\begin{array}{l}\text { Cantidad de } \\
\text { Documentos }\end{array}$} \\
\hline $\mathrm{N}$. & Universidades & $\begin{array}{l}\text { Cantidad de } \\
\text { documentos }\end{array}$ & N. & Universidades & \\
\hline 1 & $\begin{array}{l}\text { Leuphana Universität } \\
\text { Lüneburg }\end{array}$ & 18 & 1 & $\begin{array}{l}\text { Leuphana University } \\
\text { Luneburg }\end{array}$ & 14 \\
\hline 2 & $\begin{array}{l}\text { Universitat } \\
\text { Politècnica de } \\
\text { València }\end{array}$ & 8 & 2 & $\begin{array}{l}\text { Indiana University } \\
\text { Bloomington }\end{array}$ & 7 \\
\hline 3 & LUT University & 8 & 3 & $\begin{array}{l}\text { Indiana University } \\
\text { System }\end{array}$ & 7 \\
\hline 4 & $\begin{array}{l}\text { Copenhagen } \\
\text { Business School }\end{array}$ & 8 & 4 & University Of Sussex & 7 \\
\hline 5 & $\begin{array}{l}\text { Universidade da } \\
\text { Beira Interior }\end{array}$ & 7 & 5 & $\begin{array}{l}\text { Indian Institute Of } \\
\text { Management lim } \\
\text { System }\end{array}$ & 6 \\
\hline 6 & Utrecht University & 7 & 6 & $\begin{array}{l}\text { Universidade Da } \\
\text { Beira Interior }\end{array}$ & 6 \\
\hline 7 & Universität Oldenburg & 7 & 7 & $\begin{array}{l}\text { University System } \\
\text { Of Georgia }\end{array}$ & 6 \\
\hline 8 & Middlesex University & 6 & 8 & $\begin{array}{l}\text { California State } \\
\text { University System }\end{array}$ & 5 \\
\hline
\end{tabular}




\begin{tabular}{lccl}
$9 \begin{array}{l}\text { Copernicus Institute } \\
\text { of Sustainable } \\
\text { Development }\end{array}$ & 6 & 9 & $\begin{array}{l}\text { Johannes Kepler } \\
\text { University Linz }\end{array}$ \\
10 Universita del Salento & 6 & $10 \begin{array}{l}\text { Universidade Federal } \\
\text { De Santa Catarina } \\
\text { Ufsc }\end{array}$ & 5 \\
\hline
\end{tabular}

Fuente: Elaboración propia a partir de Scopus y WoS, 2021

\section{Tabla 4}

\section{Documentos más citados en WoS}

\begin{tabular}{|c|c|c|c|c|c|}
\hline \multicolumn{6}{|c|}{ Web Of Science } \\
\hline N. & Título & Autores & Editorial & Año & Citas \\
\hline 1 & $\begin{array}{l}\text { Three frames for } \\
\text { innovation policy: R\&D, } \\
\text { systems of innovation } \\
\text { and transformative } \\
\text { change }\end{array}$ & $\begin{array}{l}\text { Schot, Johan; } \\
\text { Steinmueller, W. } \\
\text { Edward }\end{array}$ & Research Policy & 2018 & 190 \\
\hline 2 & $\begin{array}{l}\text { Unlocking value for } \\
\text { a circular economy } \\
\text { through 3D printing: A } \\
\text { research agenda }\end{array}$ & $\begin{array}{l}\text { Despeisse, M. } \\
\text { Baumers, M. } \\
\text { Brown, P. } \\
\text { Charnley, F. } \\
\text { Ford, S. J. }\end{array}$ & $\begin{array}{l}\text { Technological } \\
\text { Forecasting and } \\
\text { Social Change }\end{array}$ & 2017 & 123 \\
\hline 3 & $\begin{array}{l}\text { Sustainable } \\
\text { Entrepreneurship: A } \\
\text { Convergent Process } \\
\text { Model }\end{array}$ & $\begin{array}{l}\text { Belz, Frank Martin } \\
\text { Binder, Julia } \\
\text { Katharina }\end{array}$ & $\begin{array}{l}\text { Business } \\
\text { Strategy and The } \\
\text { Environment }\end{array}$ & 2017 & 120 \\
\hline 4 & $\begin{array}{l}\text { Mapping out the } \\
\text { sharing economy: } \\
\text { A configurational } \\
\text { approach to sharing } \\
\text { business modeling }\end{array}$ & $\begin{array}{l}\text { Munoz, Pablo } \\
\text { Cohen, Boyd }\end{array}$ & $\begin{array}{l}\text { Technological } \\
\text { Forecasting and } \\
\text { Social Change }\end{array}$ & 2017 & 106 \\
\hline 5 & $\begin{array}{l}\text { The Role of Short- } \\
\text { Termism and } \\
\text { Uncertainty Avoidance } \\
\text { in Organizational } \\
\text { Inaction on Climate } \\
\text { Change: A Multi-Level } \\
\text { Framework }\end{array}$ & $\begin{array}{l}\text { Slawinski, Natalie } \\
\text { Pinkse, Jonatan } \\
\text { Busch, Timo } \\
\text { Banerjee, } \\
\text { Subhabrata Bobby }\end{array}$ & Business \& Society & 2017 & 84 \\
\hline
\end{tabular}




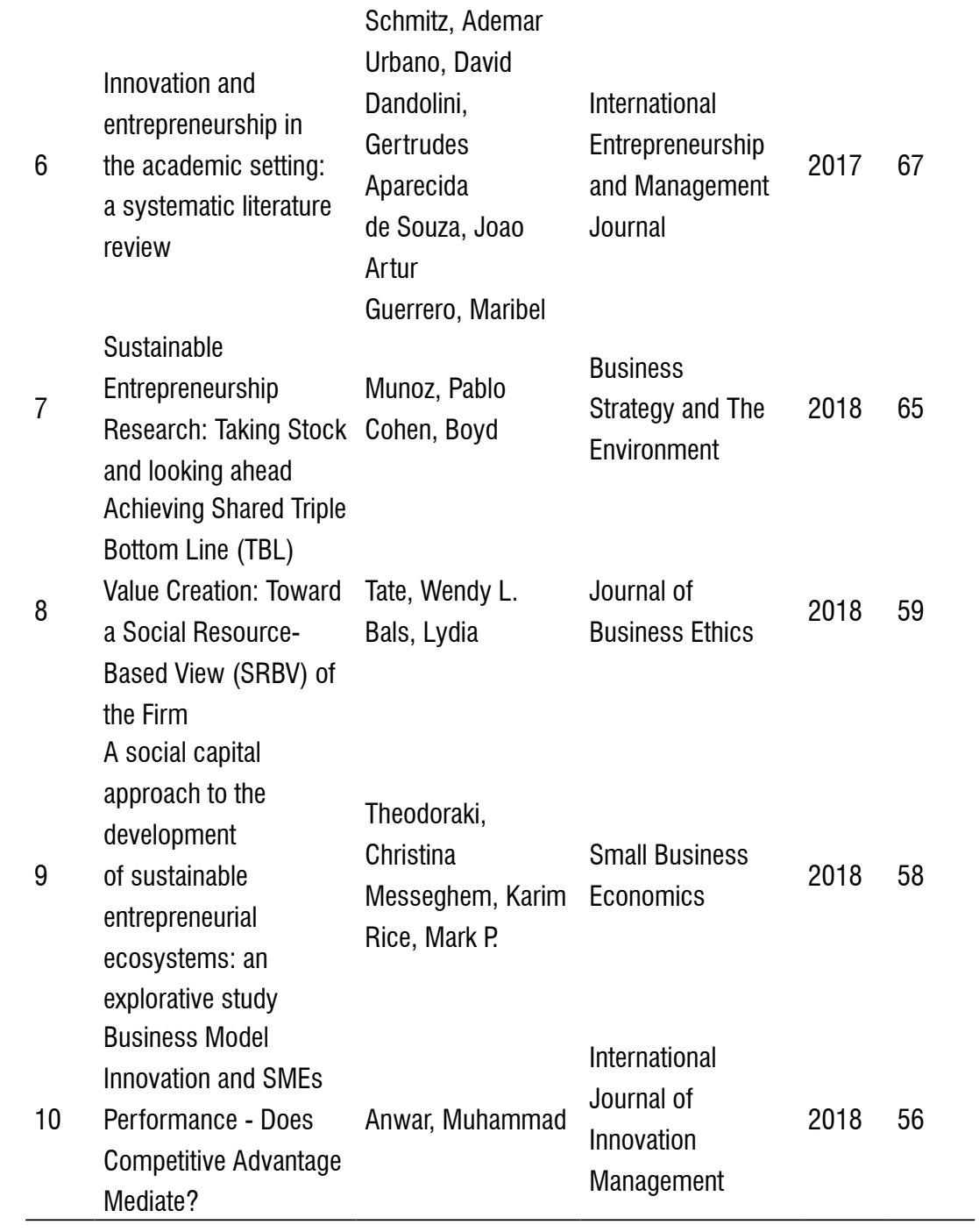

Fuente: Elaboración propia a partir de WoS, 2021.

La tabla 4 expone que en el buscador WoS el documento más citado es "Three frames for innovation policy: $R \& D$, systems of innovation and transformative change", de los autores Schot, Johan y Steinmueller, W. Edward, publicado por Research Policy en el año 2018 ha sido citado 190 veces. El segundo se titula "Unlocking value for a circular economy through 3D printing: A research agenda", de los autores Despeisse, M., Baumers, M. y Brown, P. et al., Technological Forecasting and Social Change publicado en 2017, citado 213 veces.

En la figura 3, se presentan los documentos más citados de Wos en el periodo del 2017-2021. 


\section{Figura 3}

\section{Documentos más citados por año en Wos}

Documentos más citados por año 2017-2021

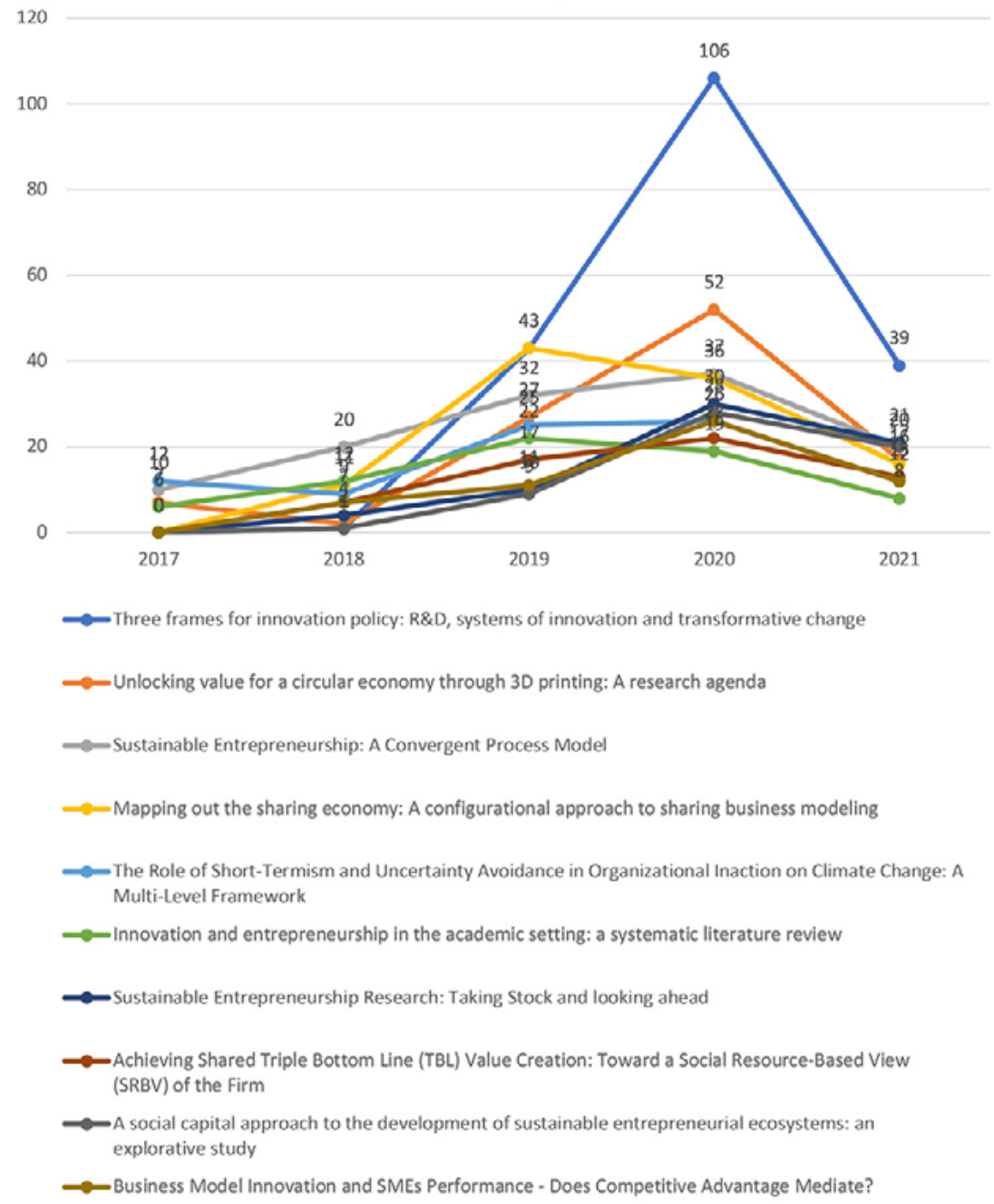

Fuente: Elaboración propia a partir de WoS (2021). 


\section{Tabla 5}

\section{Documentos más citados en Scopus}

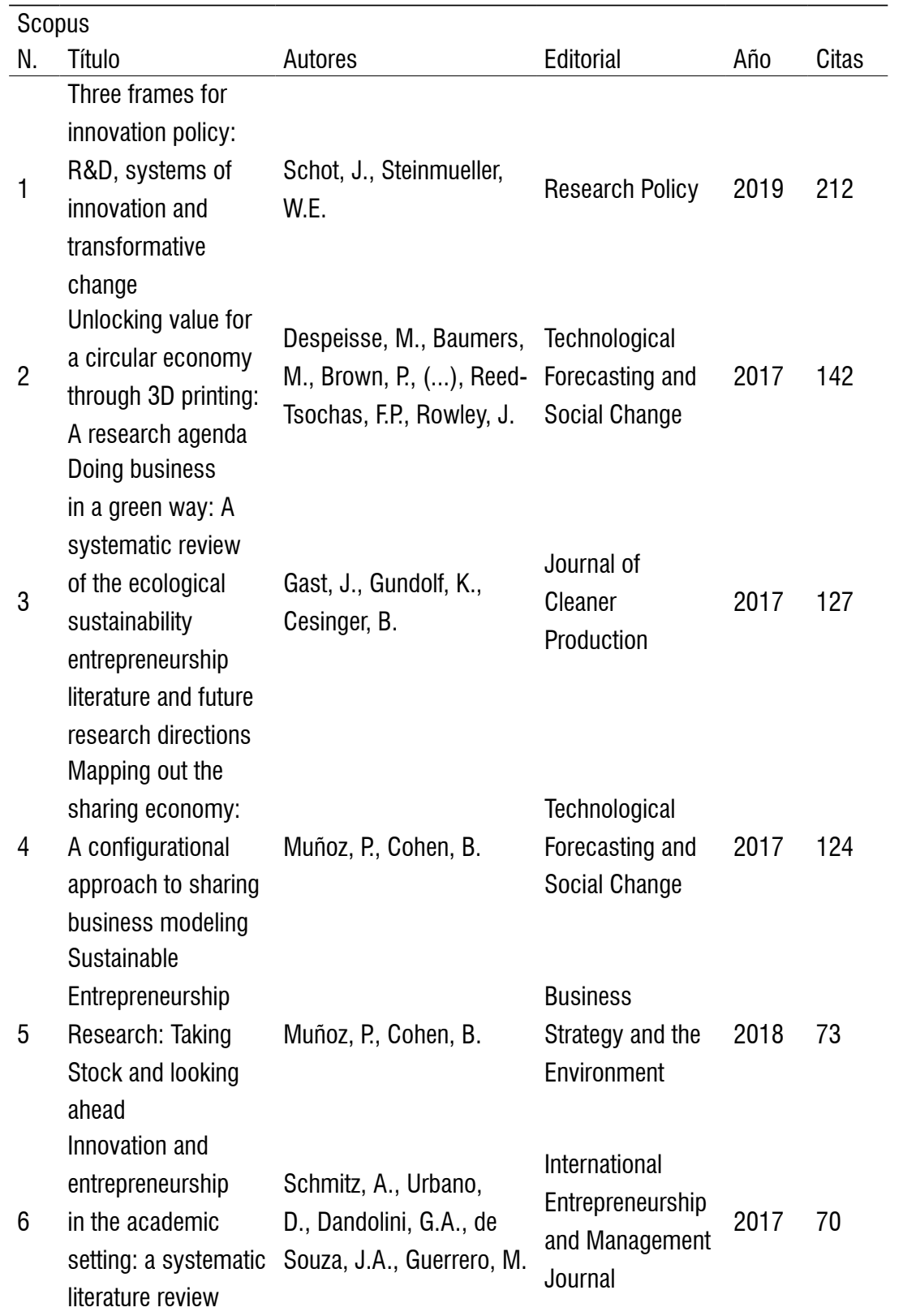




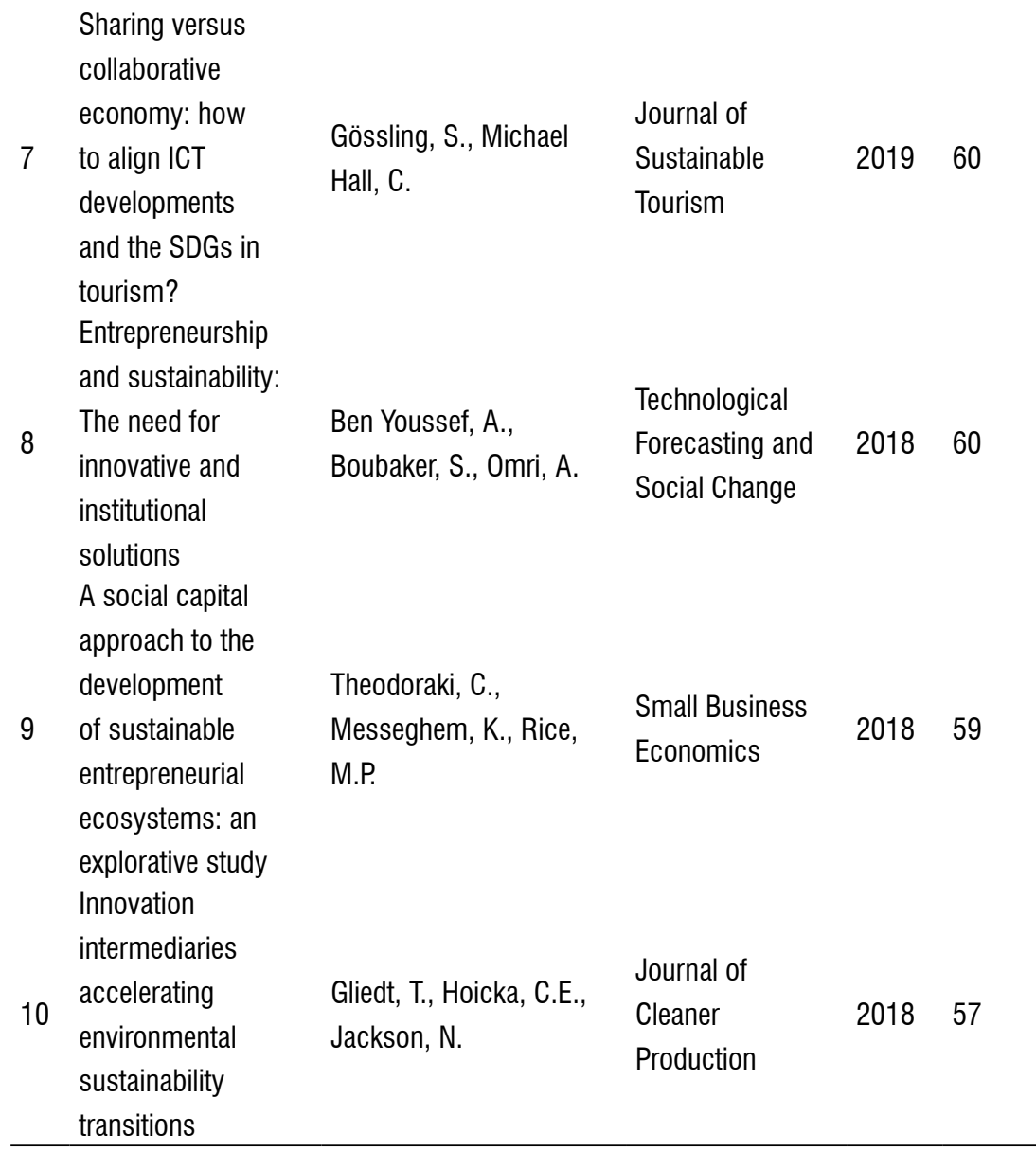

Fuente: Elaboracion propia a partir de Scopus (2021).

De acuerdo con la tabla 5 en el buscador Scopus el documento más citado esta titulado "Three frames for innovation policy: R\&D, systems of innovation and transformative change", de los autores Schot, J., y Steinmueller, W.E., publicado por la editorial Research Policy, en el año 2019, ha sido citado 212 veces. El segundo documento más citado tiene el titulo "Unlocking value for a circular economy through 3D printing: A research agenda", de los autores Despeisse, M., Baumers, M., Brown, P., (...), Reed-Tsochas, F.P., Rowley, J., publicado por la editorial Technological Forecasting and Social Change, en el año 2017, citado 142 veces. El tercer documento más citado es "Doing business in a green way: $A$ systematic review of the ecological sustainability entrepreneurship literature and future research directions", Gast, J., Gundolf, y K., Cesinger, B., publicado por la editorial Journal of Cleaner Production, se publicó en el año 2017 y tiene 127 citas. A continuación se analizan los documentos más citados por el periodo establecido 2017-2021 (ver tabla 4).

En la figura 4, se presentan los documentos más citados de Wos en el periodo del 2017-2021. 


\section{Figura 4}

\section{Documentos más citados por año en Scopus}

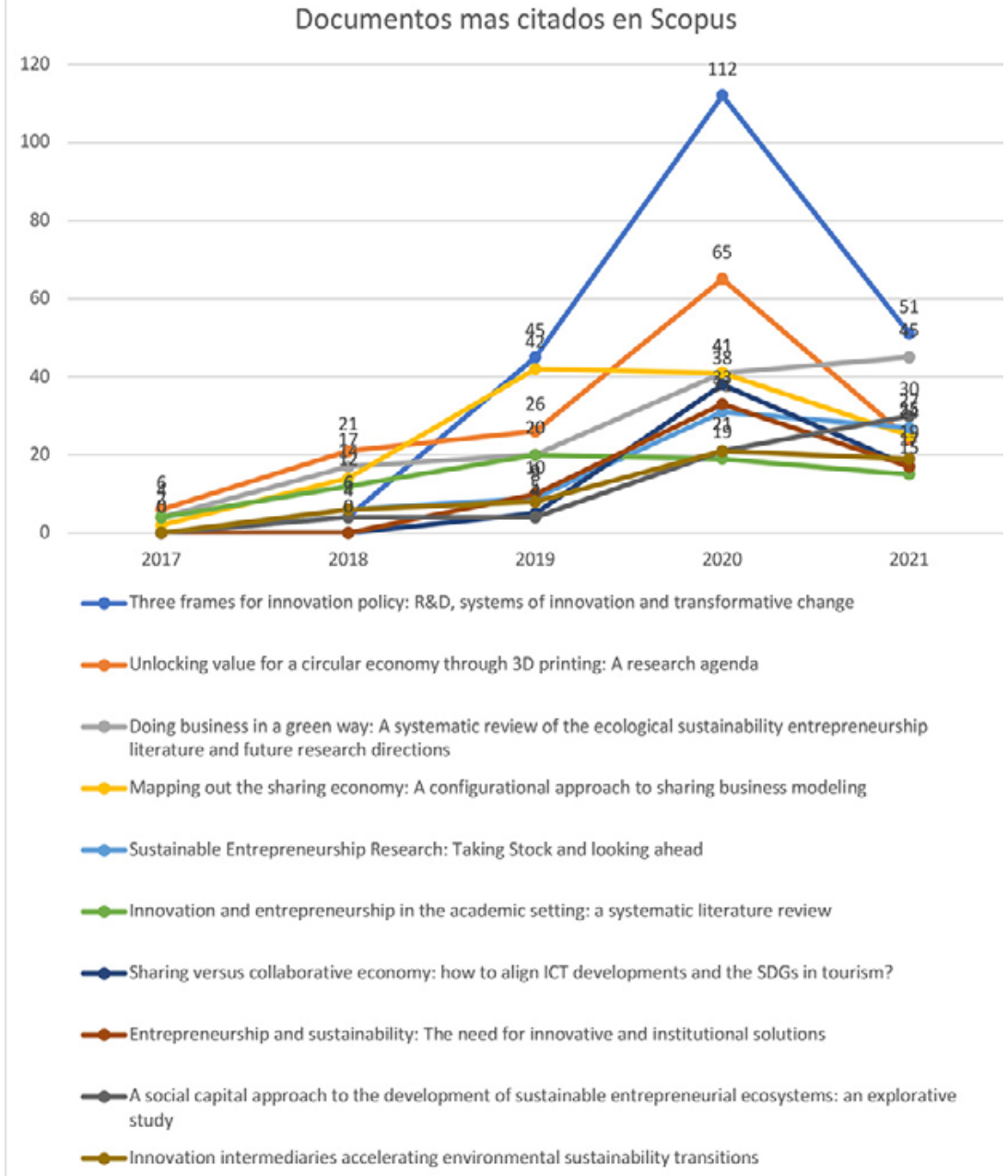

Fuente: Elaboración propia a partir de WoS, 2021. 


\section{Figura 5}

Total de citas por año en ambos buscadores

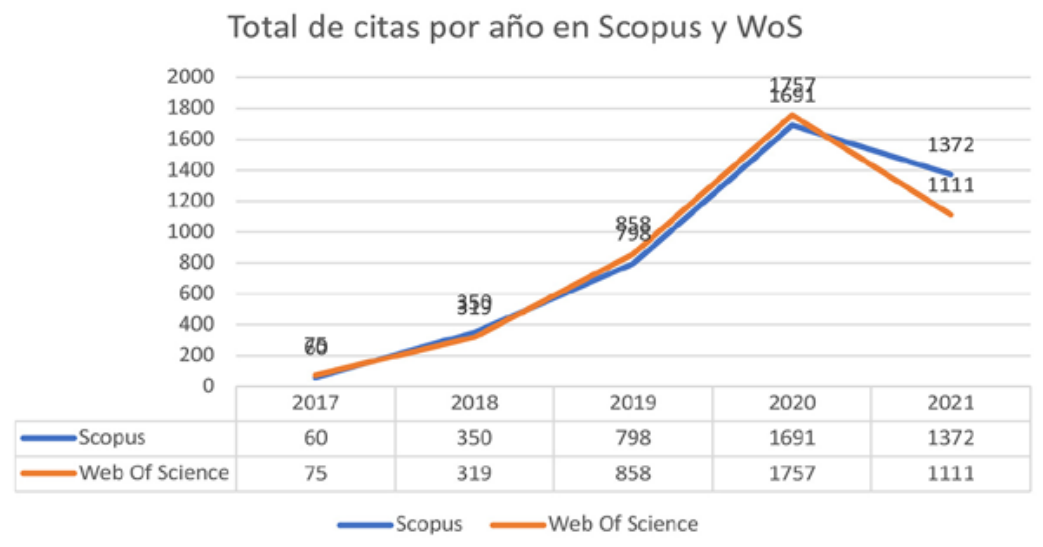

Fuente: Elaboración propia a partir de Scopus y WoS, 2021.

\section{Figura 6}

Mapa de densidad WoS

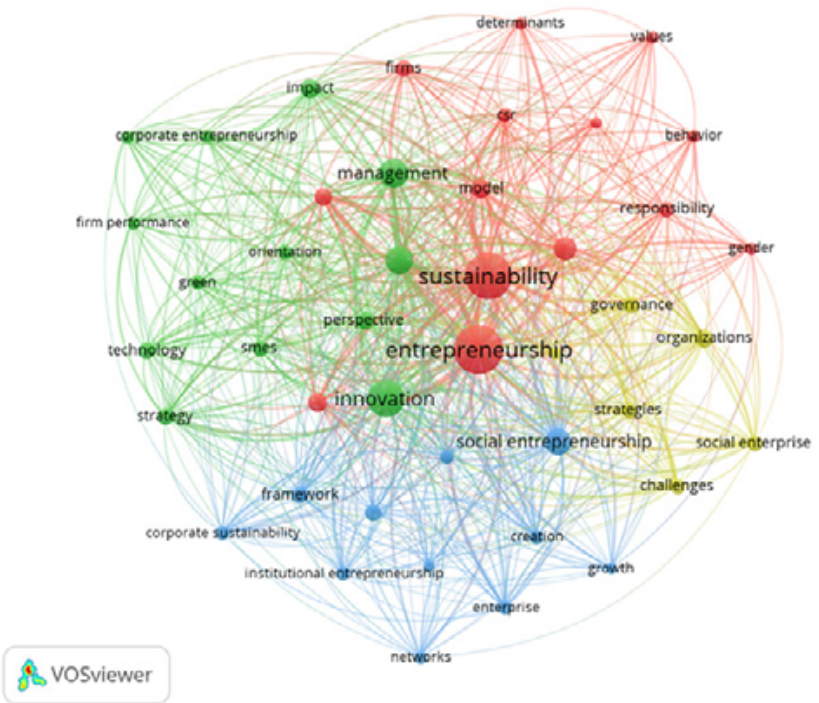

Fuente: Elaboración propia a partir de WoS, 2021.

El primer mapa de densidad fue elaborado con los documentos extraidos del buscador Web Of Science, el cual se muestra en la figura 6 . 
Los resultados obtenidos a partir de la coocurrencia en Wos y Scopus, se presentan en las siguientes tablas y destacan, pues se repitieron más de 15 veces en el total de documentos analizados.

\section{Tabla 6}

Palabras relacionadas en coocurrencia en WoS

\begin{tabular}{|c|c|c|c|}
\hline $\begin{array}{l}\text { Web Of Science } \\
\text { Grupo rojo }\end{array}$ & Grupo verde & Grupo azul & Grupo amarillo \\
\hline Sustentabilidad & Administración & $\begin{array}{l}\text { Emprendimiento } \\
\text { social e institucional }\end{array}$ & Organización \\
\hline Emprendimiento & Perspectiva & Creación & Gobernanza \\
\hline Modelo & Innovación & Empresa & Empresa social \\
\hline Responsabilidad & Pymes & Crecimiento & Desafios actuales \\
\hline Género & Estrategias & Redes colaborativas & \\
\hline Comportamiento & Verde & $\begin{array}{l}\text { Sustentabilidad } \\
\text { corporativa }\end{array}$ & \\
\hline Valor & $\begin{array}{l}\text { Resultados } \\
\text { empresariales }\end{array}$ & Marco de referencia & \\
\hline Determinantes & $\begin{array}{l}\text { Emprendimiento } \\
\text { corporativo }\end{array}$ & & \\
\hline Firmas corporativas & Impacto & & \\
\hline
\end{tabular}

Fuente: Elaboración propia a partir de WoS, 2021.

Las palabras en cada bloque están más relacionadas en el mundo académico.

\section{Tabla 7}

Palabras relacionadas en coocurrencia en Scopus

\begin{tabular}{|c|c|c|c|c|}
\hline $\begin{array}{l}\text { Scopus } \\
\text { Grupo rojo }\end{array}$ & Grupo verde & Grupo azul & Grupo amarillo & Grupo morado \\
\hline Economía & $\begin{array}{l}\text { Responsabilidad } \\
\text { social corporativa }\end{array}$ & Innovación & Sustentabilidad & $\begin{array}{l}\text { Emprendimiento } \\
\text { social }\end{array}$ \\
\hline Crowfunding & Economía circular & Educación & Emprendimiento & $\begin{array}{l}\text { Innovación } \\
\text { social }\end{array}$ \\
\hline Ecosistemas & $\begin{array}{l}\text { Emprendimiento } \\
\text { corporativo }\end{array}$ & $\begin{array}{l}\text { Modelo de } \\
\text { negocios }\end{array}$ & Negocios & Empresa social \\
\hline $\begin{array}{l}\text { Planificación } \\
\text { Ecosistemas } \\
\text { emprededores } \\
\text { Efecto social }\end{array}$ & Liderazgo & $\begin{array}{l}\text { Estrategias } \\
\text { Orientación } \\
\text { emprendedora }\end{array}$ & Pymes & \\
\hline
\end{tabular}


Revista de Investigación Aplicada en Ciencias Empresariales

\section{Figura 7}

\section{Mapa de densidad Scopus}

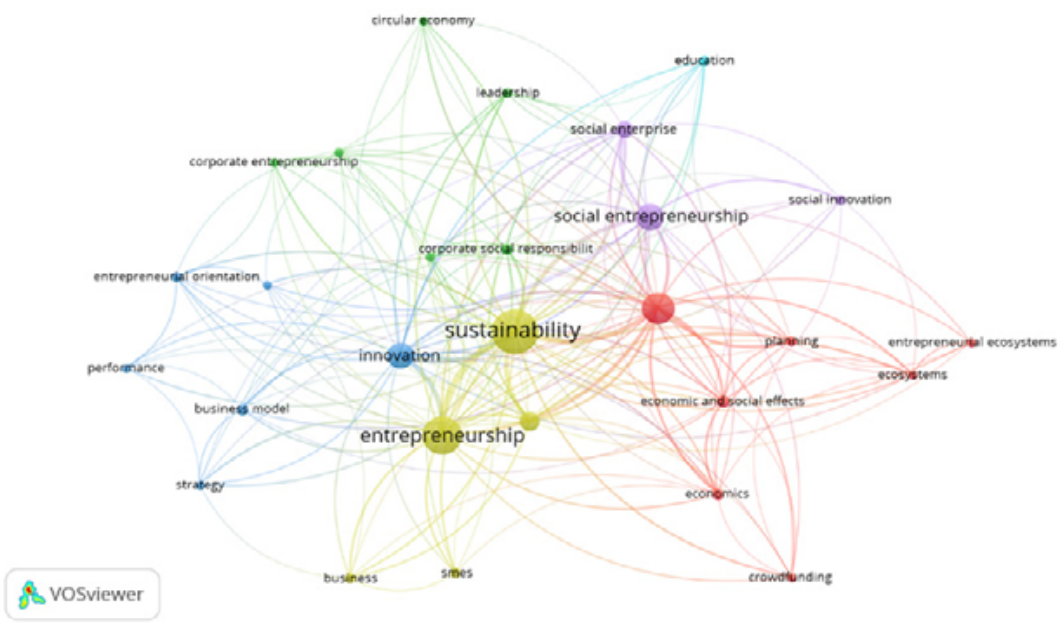

Fuente: Elaboración propia a partir de Scopus, 2021. 


\section{CONCLUSIONES}

El desgaste y el uso poco sustentable de los recursos ha causado diversos problemas ambientales y sociales alrededor del mundo. Aunado a esto el solo buscar cumplir con el objetivo económico por parte las empresas ha llevado a incrementar un uso de recursos para la elaboración de productos y servicios de corta duración de vida.

Como afirma Schot y Steinmueller (2018) el inmerso mundo competitivo que emergió a partir de la globalización del 1980, dio origen aún comportamiento consumista, quizás respaldado por las politicas de ciencias, tecnología e innovación, lo cual dichas politicas se enfocarán en la creación de vinculos, agrupaciones, redes y en estimular el aprendizaje en conjunto con elementos de sistemas y modelos empresariales para motivar el espíritu emprendedor. En contraste DiVito y Bohnsack (2017) mencionan que las empresas deben de cambiar el enfoque que han mantenido en los últimos cinco decadas que es hacer-usar-desechar, para lo cual en un economía circular se busca eliminar el concepto de desecho por el concepto de reutilizar para que el ciclo de uso sea ciclico, de esta manera las necesidades de la población serán satisfechas pero cuidando el planeta.

No obstante, en palabras de Urbaniec (2018) dice que ha emergido la inquietud de algunos grupos alrededor del mundo que buscan un equilibrio en la generación de ingresos y cuidado del medio ambiente al momento de emprender alguna idea de negocio, es ahí donde surge el emprendimiento sustentable el cual es la acción precisa de integrar en las empresas y organizaciones una orientación que considere las tres dimensiones del desarrollo sustentable el económico, social y ambiental de manera equilibrada, investigando nuevas alternativas en sus procesos de producción y uso de los productos y servicios que se desea vender.

Para finalizar con el presente estudio bibliométrico, se concluye que el emprendimiento sustentable demanda un desarrollo creativo con un pensamiento abierto que se pueda traducir en innovación empresarial. La innovación empresarial es un gran aliado en el emprendimiento sustentable, ya que incentiva y motiva a las empresas a buscar nuevas alternativas más amigables con el planeta.

Las problemáticas que causó el buscar solo el beneficio económico de manera poco amigable se ha identificado con mayor contaminación, desigualdad social y extinción de plantas y especies animales. La sustentabilidad no está peleada o es opuesto a la utilidad económica, simplemente el objetivo que integra a los modelos de negocios sustentables es cuidar los recursos antes de que desaparezcan del planeta.

Asimismo, con el emprendimiento sustentable se busca el mejoramiento desde la cadena de valor hasta el posconsumo por parte del mercado meta seleccionado. Emerge por el gran incremento de consumo poco amigable que la humanidad ha mostrado en los últimos años, no obstante, este enfoque no solo involucra a las empresas, sino también a los consumidores o clientes de dichas empresas. El 
integrar las dimensiones de la sustentabilidad conformadas por la ecológica, social y económica en los hábitos de consumo de los consumidores es un gran reto para el emprendimiento sustentable, ya que el consumo de los clientes son los que reflejan el éxito del modelo de negocio. Finalmente, invita a crear ingresos, pero sin perder de vista los aspectos sociales y ambientales que conforman el desarrollo sustentable, el canalizarse únicamente en generar ingresos han ocasionado que se pierda de vista estas dos dimensiones.

Este nuevo enfoque demanda un pensamiento no convencional, al integrar aspectos sustentables, indagar en nuevos procesos y reconsiderar las acciones que están ejerciendo, representando un reto importante, pero no imposible.

\section{Futuras líneas de investigación}

El implementar enfoques sustentables en los modelos de negocios abre la posibilidad de una nueva exploración en nuevos procesos para la producción de productos y servicios los cuales no afecten al medio ambiente una vez finalizado su ciclo de vida, al volver al lugar de donde fueron extraídos que fue la tierra, no dañará los ecosistemas, permitiendo que se reutilice nuevamente, brindado la posibilidad de que otras generaciones lo usen, además, el promover los modelos de negocios sustentables despertará la motivación de consumo de posibles mercados específicos que muestran esa preferencia por consumir de manera sustentable y amigable con su entorno, tanto en lo social, económico y ambiental. 


\section{REFERENCIAS BIBLIOGRÁFICAS}

AL OMOUSH, K., AL-QIREM, R., \& AL HAWATMAH, Z. (2017). The degree of e-business entrepreneurship and long- term sustainability: an institutional perspective . Inf Syst E-Bus Manage, 29-56.

AYALA-PICAZO, M. (2017). Estudio bibliométrico de la revista Anales Médicos (1955-2015). Parte 2, análisis de la producción científica. Revista Anales Médico, 62(1), 5-9.

AZAM R0OMI, M., SAIZ-ALVAREZ, J., \& CODURAS, A. (2021). Measuring Sustainable Entrepreneurship and Eco-Innovation: A Methodological Proposal for the Global Entrepreneurship Monitor (GEM). Sustainability .

BELZ, F., \& BINDER, J. (2017). Sustainable Entrepreneurship: A Convergent Process Model. Business Strategy and the Environment, 1-17.

BEN YOUSSEF, A., BOUBAKER, S., \& OMRI, A. (2018). Entrepreneurship and sustainability: The need for innovative and institutional solutions. TECHNOLOGICAL FORECASTING AND SOCIAL CHANGE, 232-241.

CARAYANNIS, E., GRIGOROUDIS, E., DEL GIUDICE, M., DELLA PERUTA, M., \& SINDAKIS, S. (2017). An exploration of contemporary organizational artifacts and routines in a sustainable excellence context. Journal of Knowledge Management, 35-56.

CHU, F., ZHANG, W., \& JIANG, Y. (2021). How Does Policy Perception Affect Green Entrepreneurship Behavior? An Empirical Analysis from China. Discrete Dynamics in Nature \& Society, 9.

DEMIREL, P., LI, Q., RENTOCCHINI, F., \& TAMVADA, J. (2019). Born to be green: new insights into the economics and management of green entrepreneurship. SMALL BUSINESS ECONOMICS, 759-771.

DIVITO, L., \& BOHNSACK, R. (2017). Entrepreneurial orientation and its effect on sustainability decision tradeoffs: The case of sustainable fashion firms. JOURNAL OF BUSINESS VENTURING, 569-587.

FERREIRA LEITE, M., SIDRIM DE FIGUEIREDO MENDONÇA, F., MAIA TAVARES, F., ALENCAR JULIÃO CABRAL, N., \& ARAÚJO MAIA, E. (2021). GEOPRODUTOS EM COMUNIDADES TURÍSTICAS PARA 0 DESENVOLVIMENTO SUSTENTÁVEL E EMPREENDEDORISMO SOCIAL: UM ESTUDO DE CASO. Revista Producao Online, 913-929.

GAST, J., GUNDOLF, K., \& CESINGER, B. (2017). Doing business in a green way: A systematic review of the ecological sustainability entrepreneurship literature and future research directions. Journal of Cleaner Production, 44-56. 
GUTA, G., VHUDZI, G., \& CHAZOVACHII, B. (2017). Sustainability of Rural Entrepreneurship as a Livelihood Strategy in Zaka District, Zimbabwe. Bangladesh e-Journal of Sociology.

HALL, J., DANEKE, G., \& LENOX, M. (2010). Sustainable development and entrepreneurship: Past contributions and future directions. JOURNAL OF BUSINESS VENTURING, 439-448.

HORISCH, J., KOLLAT, J., \& BRIEGER, S. (2017). What influences environmental entrepreneurship? A multilevel analysis of the determinants of entrepreneurs' environmental orientation. SMALL BUSINESS ECONOMICS, 47-69.

HRIVNÁK, M., MORITZ, P., \& CHRENEKOVÁ, M. (2021). What Kept the Boat Afloat? Sustainability of Employment in Knowledge-Intensive Sectors Due to Government Measures during COVID-19 Pandemic. Sustainability, 8441.

J00 LEE, H. (2019). What Factors Are Necessary for Sustaining Entrepreneurship? Sustainability.

LITTLEWOOD, D., \& HOLT, D. (2018). Social Entrepreneurship in South Africa: Exploring the Influence of Environment. Business and Society, 525-561.

LIU, H., KULTUREL-KONAK, S., \& KONAK, A. (2021). Key Elements and Their Roles in Entrepreneurship Education Ecosystem: Comparative Review and Suggestions for Sustainability. Sustainability .

LUU, T. (2017). CSR and organizational citizenship behavior for the environment in hotel industry The moderating roles of corporate entrepreneurship and employee attachment style. INTERNATIONAL JOURNAL OF CONTEMPORARY HOSPITALITY MANAGEMENT, 2867-2900.

MUÑOZ, P., \& COHEN, B. (2018). Sustainable Entrepreneurship Research: Taking Stock and looking ahead. Business Strategy and the Environment, 300-322.

MURALIDHARAN, E., \& PATHAK, S. (2018). Sustainability, Transformational Leadership, and Social Entrepreneurship. Sustainability.

PIETRZAK, M., BALCERZAK, A., GAJDOS, A., \& ARENDT, L. (2017). ENTREPRENEURIAL ENVIRONMENT AT REGIONAL LEVEL: THE CASE OF POLISH PATH TOWARDS SUSTAINABLE SOCIO-ECONOMIC DEVELOPMENT. ENTREPRENEURSHIP AND SUSTAINABILITY ISSUES, 190-203.

SAEBI, T., FOSS, N., \& LINDER, S. (2019). Social Entrepreneurship Research: Past Achievements and Future Promises. JOURNAL OF MANAGEMENT, 70-95. 
SCHOT, J., \& STEINMUELLER, W. (2018). Three frames for innovation policy: R\&D, systems of innovation and transformative change. Research Policy, 1554-1567.

SCHULENKORF, N. (2017). Managing sport-for-development: Reflections and outlook. Sport Management Review, 243-251.

SCHULENKORF, N. (2017). Managing sport-for-development: Reflections and outlook. SPORT MANAGEMENT REVIEW, 243-251.

SKURATOVYCH, I., LYTVYN, N., PANOVA, I., OVCHARENKO, I., \& HRYHOREVSKA, 0. (2021). EFFICIENCY OF USING THE POTENTIAL OF SMALL BUSINESS IN ENSURING SUSTAINABLE ECONOMIC GROWTH. ENTREPRENEURSHIP AND SUSTAINABILITY ISSUES, 742-756.

SLAWINSKI, N., PINKSE, J., BUSCH, T., \& BANERJEE, S. (2017). The Role of Short-Termism and Uncertainty Avoidance in Organizational Inaction on Climate Change: A Multi-Level Framework. BUSINESS \& SOCIETY, 253282.

SOO SUNG, C., \& PARK , J. (2018). Sustainability Orientation and Entrepreneurship Orientation: Is There a Tradeoff Relationship between Them? Sustainability, 1-15.

STUBBS, W. (2017). Sustainable Entrepreneurship and B Corps. BUSINESS STRATEGY AND THE ENVIRONMENT, 331-344.

THEODORAKI, C., MESSEGHEM, K., \& RICE, M. (2018). A social capital approach to the development of sustainable entrepreneurial ecosystems: an explorative study. SMALL BUSINESS ECONOMICS, 153-170.

URBANIEC, M. (2018). Sustainable Entrepreneurship: Innovation-Related Activities in European Enterprises. POLISH JOURNAL OF ENVIRONMENTAL STUDIES, 1773-1779.

VÁSQUEZ-BERNAL, 0., \& MOSQUERA-LAVERDE, W. (2021). Analysis of the sustainability management model in the productivity of small and medium enterprises. Case study in the city of Bogotá D.C. Proceedings of the International Conference on Industrial Engineering \& Operations Management, 302/307.

YANG, S., WANG, H., WANG, Z., KOONDHAR, M., \& KONG, R. (2021). The Nexus between Formal Credit and E-Commerce Utilization of Entrepreneurial Farmers in Rural China: A Mediation Analysis. JOURNAL OF THEORETICAL AND APPLIED ELECTRONIC COMMERCE RESEARCH, 900-921. 